\title{
CIMENTOS DE SILICATO, FABRICADOS NO BRASIL, FACE ÀS NORMAS INTERNACIONAIS

\section{S I N $\mathbf{O}$ P $\mathbf{S} \quad \mathbf{E}$}

Verificação do comportamento, face à normas internacionais, dos dois únicos cimentos de silicato de uso odontológico fabricados no Brasil. Observado o atendimento às normas, exceptuando-se a resistência à compressão, que nos pareceu baixa no silicato de marca Astralit. Trabalhos desta natureza propiciam a melhoria dos produtos, na indístria nacional de materiais dentários.

\section{INTRODUÇ̃̃O}

Em março de 1971 foi apresentado à Federação Dentária Internacional, pelo grupo de trabalho «ISO/TC 106 / WG 1 - Filling Materials», através de seu Documento 62, o primeiro Projeto de Normas para silicatos e silico-fosfatos encapsulados.

No Brasil ainda não se fabricam silicatos com este tipo de acondicionamento, mas o fato em si, da exis-

\author{
Prof. Léo Werner Süffert * \\ Prof. Ennio Pessôa ** \\ Dr. Airton Teixeira Brasil ***
}

tência de um Projeto de Normas, em âmbito internacional, diz bem da importância e da aceitação cada vez maior que terá por parte da odontologia moderna, este tipo de acondicionamento.

Para os cimentos de silicato acondicionados em frascos comuns já existem normas há muito estabelecidas e aceitas por diversos países, seja através de suas Associações Odontológicas Nacionais,, como é o caso da American Dental Association 1,2 e outras, seja através de grupos especializados, como é o caso do Grupo Brasileiro de Materiais Dentários 3 (Comitê da Associação Brasileira de Normas Técnicas - ABNT), ou seja ainda através de Associações Internacionais da classe Odontológica, como é o caso da Federation Dentaire International ${ }^{4}$, ou de Padronização, como é o caso da ISO (International Standards Organization) ${ }^{5}$

Esta última organização, a Associação Internacional de Padrões, é o órgão internacional oficial, ao qual esstão filiadas todas as Associações Nacionais de Normas.

\footnotetext{
- Professor Catedrático de Materiais Dentários, Faculdade de Odontologia, UFRGS

* Professor Adjunto de Materiais Dentários, Faculdade de Odontoolgia, UFRGS

** Estagiário da Disciplina de Materiais Dentários, Faculdade de Odontologia, UFRGS Bolsa de Aperfeiçoamento do Conselho de Pesquisas da UFRGS
} 


\section{OBJE'TIV0}

O objetivo do presente trabalho é o de verificar como se comportam, face às normas acima referidas, os dois únicos cimentos de silicato de uso odontológico fabricados no Brasil.

\section{MATERIAIS, MÉTODOS DE EN- SAIO E RESUL'TADOS OBTIDOS}

As duas marcas de silicato estudadas podem ser observadas na Fig de $\mathrm{N}:$ 1. Observe-se que escolhemos a mesma cor em ambos os casos, particularmente por se tratar da cor mais largsmente empregada.

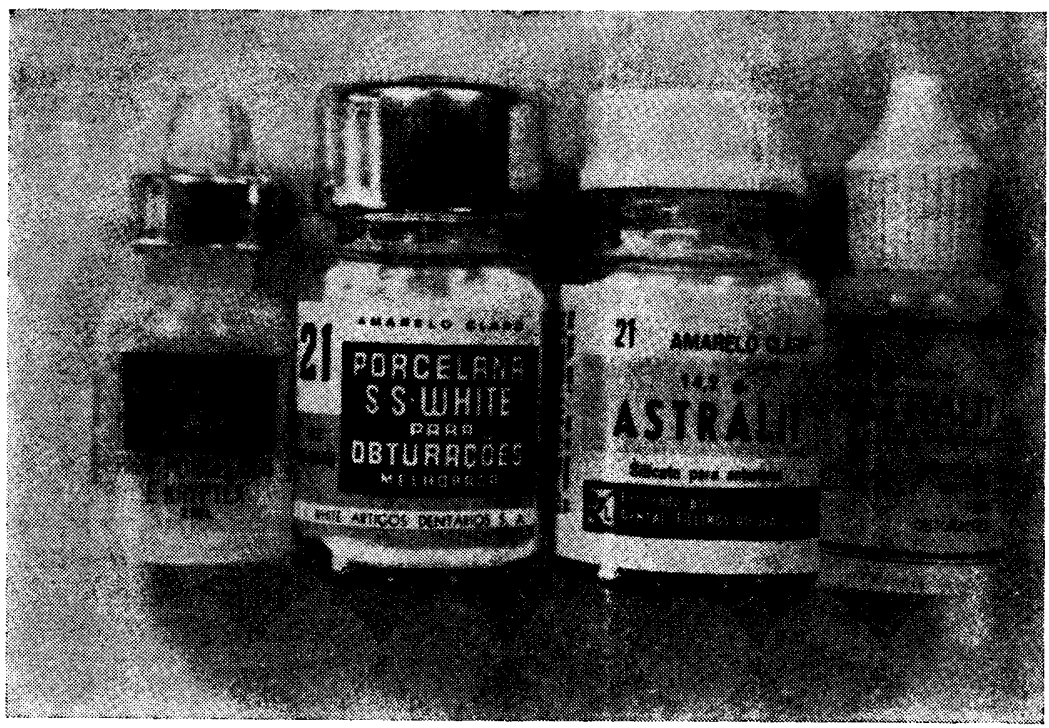

Figura $N: 1$

\subsection{GENERALIDADES}

Antes de efetuarmos qualquer tipo de exame, procedemos como indicado em 4.1 .1 e 4.1 .2 das normas 2,3 em relação à mistura de líquidos $\mathrm{e}$ mistura de pós. O frasco, especialmente adatado, submetido à rotação em torno de seu menor eixo, à uma velocidade de 25 revoluções por mi- nuto, durante duas horas, nos permitia uma excelente mistura do material coletado de diversos frascos de embalagem original. (Fig de $\mathbf{N} ! 2$ )

Ao procedermos com a inspeção visual, constatamos em ambas as marcas, o preenchimento quanto aos requisitos $3.1,3.2 .1,3.3 .1,3.4 .1$, $3.5 .1,3.8,4.3 .5$ e 5.1

R. Fac. Odont. P. A. 


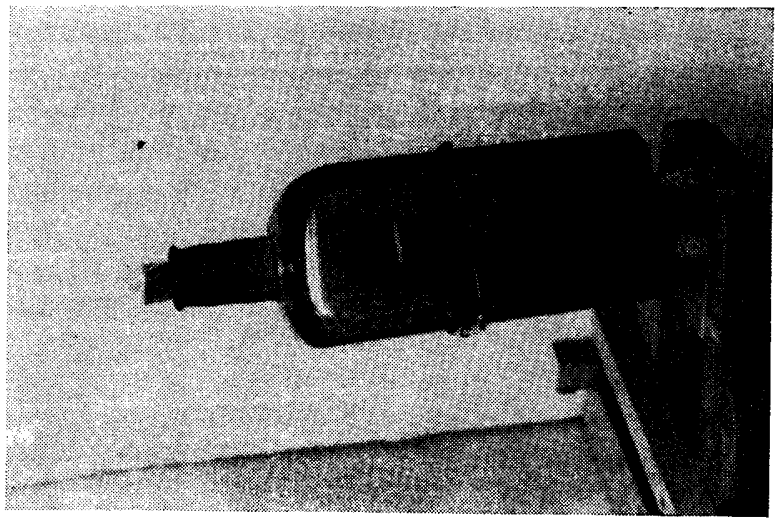

Figura $N^{\circ} 2$

\subsection{CONSISTENCIA PADR̃̃o}

A quantidade de pó, previamente dicação da temperatura (Fig de $\mathrm{N}^{\mathbf{0}} \mathbf{3}$ ). Procedia-se em seguida à sua mapesado em balança sensível ao $\mathrm{mg}$, nipulação, de acordo com 4.3.1 e bem como a o do líquido $(0,40 \mathrm{ml}) \quad 4.3 .2$ afim de verificar qual a quaneram colocados em uma lage com in- tidade de pó necessária à cada mar-

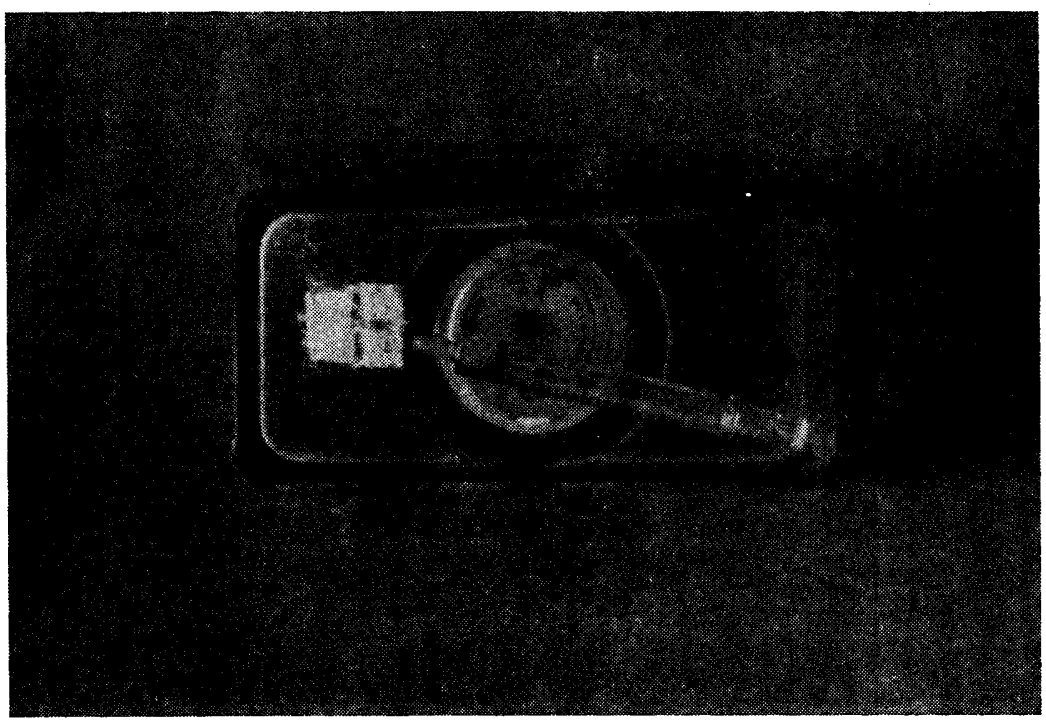

Figura $N^{9} 3$

R. Fac. Odont. P. A. 
ca silicato, para nos indicar a «consistência padrão».

Na Fig de No 4, podemos observar o «disco» resultante da aplicação de uma carga de $2.500 \mathrm{G}$ sobre um volume de $0,50 \mathrm{ml}$ do cimento misturado.

Realizados $10^{\circ}$ ensaios com cada marca de silicato e obtido de cada ensaio uma média de duas leituras, chegamos ao seguinte resultado final:

Percebe-se, pois, que podemos incorporar um maior volume de pó à uma mesma quantidade de líquido no caso do silicato $\mathrm{S}$. S. White.

Possivelmente sua granulometria seja bem menor do que no caso do Astralit.

\author{
Temperatura ambiente: \\ Umidade relativa: \\ Proporção pó/líquido: \\ Média do disco em $\mathrm{mm}$ :
}

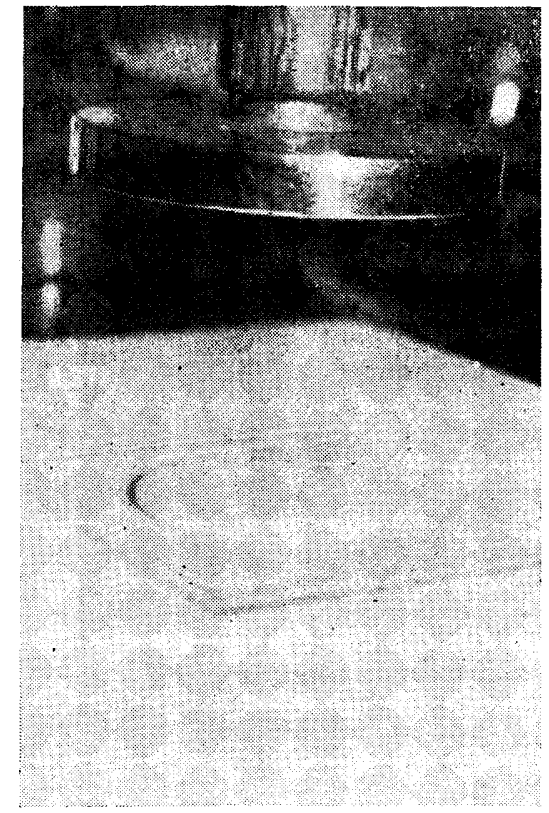

Figura $N ?+$

S. S. WHITE

$$
\begin{gathered}
\text { ASTRALIT } \\
20^{\circ} \mathrm{C} \\
66 \% \\
1,00 \mathrm{~g} / 0,40 \mathrm{ml} \\
24,8 \mathrm{~mm}
\end{gathered}
$$

$21^{\circ} \mathrm{C}$

$55 \%$

$1,40 \mathrm{~g} / 0,40 \mathrm{ml}$

$24,9 \mathrm{~mm}$

\subsection{SOLUBILIDADE E DESINTE- GRACX̃̃O}

Neste ensaio é de capital importância a pesaçem com alta precisão dos corpos de prova, o que é feito mediante um frasco pesa-filtro, (Figura 5) em balança especial. Decorrido o espaço de tempo de 24 horas, durante o qual o silicato sofre as conseqüências de sua solubilidade e se desintegra em maior ou menor grau, remove-se os corpos de prova do frasco pesa-filtro e leva-se por diversas vezes à estufa (Fig de No6) inicialmente com temperatura ligeiramente abaixo de $100^{\circ} \mathrm{C}$ e posteriormente à $150^{\circ} \mathrm{C}$, até pesagem constante.

Os resultados obtidos com cinco ensaios de cada marca, são apresentados à seguir: 

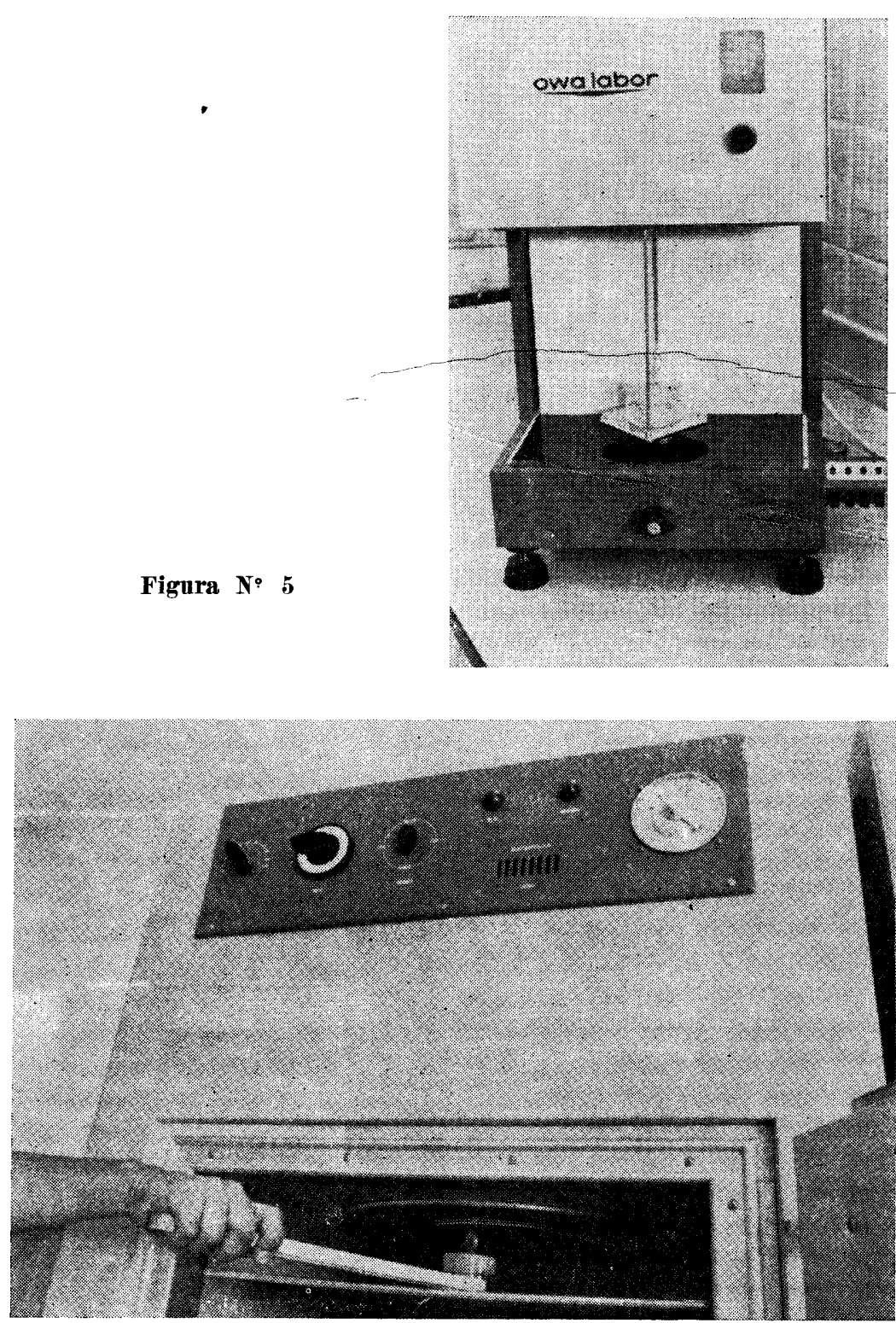

Figura $N^{0} 6$

R. Fac. Odont. P. A. 


\subsubsection{Resultados com silicato S. S. White:}

\section{1? Ensaio}

Temperatura: $22^{\circ} \mathrm{C}$

Umidade relativa: $45 \%$

Peso inicial do frasco: ........

$49,8728 \mathrm{~g}$ (média de 2 pesagens)

Peso final do frasco: ..........

Aumento de peso:

$49,8781 \mathrm{~g}$ (média de 5 pesagens)

Peso do corpo de prova: ........

$0,0053 \mathrm{~g}$

Percent. desintegração: $\ldots \ldots \ldots(0,0053 \div 1,1958) \times 100=0,44 \%$

\section{2: Ensaio}

Temperatura: $23{ }^{\circ} \mathrm{C}$

Peso inicial do frasco: ..........

Peso final do frasco:

Umidade relativa: $65 \%$

$49,8750 \mathrm{~g}$ (média de 2 pesagens)

49,8819 g (média de 5 pesagens)

Aumento de peso:

$0,0069 \mathrm{~g}$

$1,4096 \mathrm{~g}$

Peso do corpo de prova: ........

Percent. desirtegração: $\ldots \ldots \ldots(0,0069 \div 1,4096) \times 100=0,48 \%$

\section{3: Ensaio}

Temperatura: $23 \% \mathrm{C}$

Umidade relativa: $65 \%$

Peso inicial do frasco: .........

$47,7215 \mathrm{~g}$ (média de 2 pesagens)

Peso final do frasco: ......... 47,7275 g (média de 5 pesagens)

Peso do corpo de prova: ...... $1,2133 \mathrm{~g}$

Percent. desintegração: $\ldots \ldots \ldots(0,0060 \div 1,2133) \times 100=0,49 \%$

\section{$4^{\circ}$ Ensaio}

Temperatura: $23^{\circ} \mathrm{C}$

Peso inicial do frasco: .........

Umidade relativa: $54 \%$

Peso final do frasco: .........

Aumento de peso: ...........

Peso do corpo de prova: .......

Percent. desintegração: ........

\section{5' Ensaio}

Temperatura: $21^{\circ} \mathrm{C}$

Peso inicial do frasco: ........

Peso final do frasco: $\ldots \ldots \ldots \ldots$

Aumento de peso: ............

Peso do corpo de prova: .......

$49,8700 \mathrm{~g}$ (média de 2 pesagens)

$49,8812 \mathrm{~g}$ (média de 5 pesagens)

$0,0112 \mathrm{~g}$

$1,8903 \mathrm{~g}$

$(0,0112 \div 1,8903) \times 100=0,59 \%$

Umidade relativa: $45 \%$

$49,8762 \mathrm{~g}$ (média de 2 pesagens)

49,8832 g (média de 5 pesagens)

$0,0070 \mathrm{~g}$

$1,1597 \mathrm{~g}$

$(0,0070 \div 1,1597) \times 100=0,60 \%$

M D I A : $\quad 0,52 \%$

R. Fac. Odont. P. A.

$13 / 14: 137-152,1971 / 1972$ 
3.3.2 Resultados com o silicato As tralit:

\section{Ensaio}

Temperatura : $22 \% \mathrm{C}$

Peso inicial do frasco: $\ldots \ldots \ldots \ldots$

Peso final do frasco: .........

Aumento de peso:

Peso do corpo de prova: .......

Percent. desintegração: .......

\section{- Ensaio}

Temperatura: $23^{\circ} \mathrm{C}$

Peso inicial do frasco: ........

Peso final do frasco: ..........

Aumento de peso: ...........

Peso do corpo de prova: ......

Percent. desintegração: .......

\section{Ensaio}

Tomporatura: $21^{\circ} \mathrm{C}$

Peso inicial do frasco: . . . . . . . .

Peso final do frasco: .........

Aumento de peso: ...........

Peso do corpo de prova: ......

Percent. desintegração: .......

\section{1: Ensaio}

Temperatura: $22^{\circ} \mathrm{C}$

Peso inicial do frasco: ........

Peso final do frasco: ..........

Aumento de peso: ............

Peso do corpo de prova: .......

Percent. desintegração: .......

\section{Ensaio}

Temperatura: $23^{\circ} \mathrm{C}$

Peso inicial do frasco: ........

Peso final do frasco: .........

Aumento de peso: ...........

Peso do corpo de prova: .......

Percent. desintegração: .......

o máximo de solubilidade e desintegração permíssivel, segundo as nor-

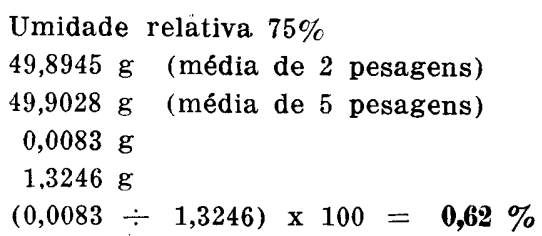

Umidade relativa: $50 \%$

$49,8725 \mathrm{~g}$ (média de 2 pesagens)

$49,8837 \mathrm{~g}$ (média de 5 pesagens)

$0,0112 \mathrm{~g}$

$1,3246 \mathrm{~g}$

$(0,0112 \div 1,3246) \times 100=0,84 \%$

Umidade relativa: $65 \%$

$47.7188 \mathrm{~g}$ (média de 2 pesagens)

$47,7316 \mathrm{~g}$ (média de 5 pesagens)

$0,0128 \mathrm{~g}$

$0,4833 \mathrm{~g}$

$(0,0128 \div 1,4833) \times 100=0,86 \%$

Umidade relativa: $47 \%$

$47,7194 \mathrm{~g}$ (média de 2 pesagens)

$47,7308 \mathrm{~g}$ (média de 5 pesagens)

$0,0114 \mathrm{~g}$

$1,3098 \mathrm{~g}$

$(0,0114 \div 1,3098) \times 100=0,87 \%$

Umidade relativa: $65 \%$

$49,8736 \mathrm{~g}$ (média de 2 pesagens)

$49,8883 \mathrm{~g}$ (média de 5 pesagens)

$0,0147 \mathrm{~g}$

$1,2369 \mathrm{~g}$

$(0,0147 \div 1,2369) \times 100=1,18 \%$

M D I A : $\quad \mathbf{0 , 8 7 4 \%}$

mas, é de 1,0\% em 24 horas. Neste sentido verificamos que ambos os 
materiais são satisfatórios.

A diferença entre os dois materiais, no entanto, é estatisticamente significativa ao nível de $.01 \mathrm{em}$ favor do silicato $\mathbf{S}$. S. White, cuja solubilidade é de $0,52 \%$ ao passo que a do silicato Astralit é de 0,87\%.

\section{$\begin{array}{rrrrrrrrrrr}3.4 & 0 & \text { P } & \text { A } & \text { C } & \text { I } & \text { D } & \text { A } & \text { D } & \text { E * } & *\end{array}$}

$O$ índice de opacidade para os cimentos de silicato, deve situar-se, segundo às normas, entre o valor mínimo de 0,35 e o valor máximo de $0,55 \mathrm{em}$ relação à $\mathrm{C} 0.70$.

Os resultados que encontramos, 0,3196 para o silicato $\mathrm{S}$. S. White e 0,3057 para o silicato Astralit demonstram em primeiro lugar, que a diferença entre os dois materiais não é estatisticamente significativa ao ní. vel de .01.
Em segundo lugar, percenc se que nenhum dos dois materiais preenche as normas neste sentido.

Alertanros, no entanto, para o fato de que estes resultados não devem ser aceitos sem uma análise mais minunciosa, a qual foi motivo de outra pesquisa, publicada nesta mesma revista. 6

\subsection{RESISTENCIA A COMPRES- SÃO}

Tanto na elaboração dos corpos de prova, como na execução do ensaio propriamente dito (Fig. 7), seguimos as recomendações do item 4.3.4 das Normas, exceto quanto ao número de corpos de nrova, que no caso foi de 50, ou seja: 5 séries de 5 corpos de prova para cada silicato ensaiado.

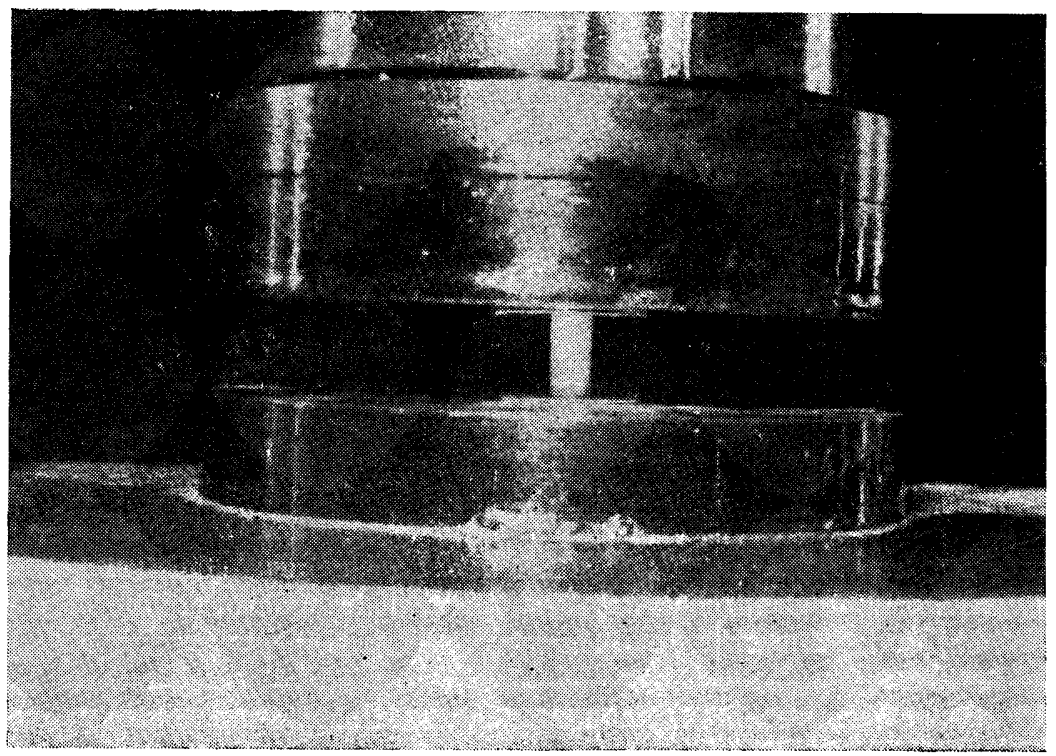

Figura $\mathrm{N} \%$

R. Fac. Odont. P. A. 
3.5 1 Resultados com o silicato S. S. White:

Temperatura $23^{\circ} \mathrm{C}$

Cargá de ruptura (kg) Esforço $\left(\mathrm{kg} / \mathrm{cm}^{2}\right) \quad$ Média

$1^{9}$ Ensaio

$2^{o}$ Ensaio

39 Ensaio

4? Ensaio

5. Ensaio

$\begin{array}{ll}440 & 1.556 \\ 460 & 1.627 \\ 480 & 1.698 \\ 460 & 1.627 \\ 430 & 1521\end{array}$

450

420

440

430

410

Umidade relativa: $66 \%$

1.605

1.592

1.486

1.556

1.521

1.450

1.521

2.122

1.910

1.769

1.627

1.592

1.804

1.698

1.663

1.698

1.627

1.521

1.641

430

1.486

1.521

1.592

1.556

1.627

1.556

460

$1625 \mathrm{~kg} / \mathrm{cm}^{2}$ 
3.5.2 Resultados con o silicato Astralit:

Temperatura $23^{\circ} \mathrm{C}$

Umidade relativa: $59 \%$

Carga de ruptura (kg) Esforço $\left(\mathrm{kg} / \mathrm{cm}^{2}\right) \quad$ Média

$1^{9}$ Ensaio

$\begin{array}{ll}440 & 1.415 \\ 330 & 1.167 \\ 320 & 1.132 \\ 350 & 1.238 \\ 380 & 1.344\end{array}$

1.259

2o Ensaio

340

1.203

370

1.309

350

1.238

360

1.273

380

1.344

1.273

3 Ensaio

380

1.344

380

1.344

380

1.344

400

1.415

390

1.380

1.365

$4^{\text {9 }}$ Ensaio

370

1.309

380

1.344

360

1.273

350

1.238

380

1.344

1.301

5? Ensaio

$\begin{array}{ll}390 & 1.380 \\ 340 & 1.203 \\ 350 & 1.238 \\ 330 & 1.167 \\ 320 & 1.132\end{array}$

1.224

Média geral dos 5 ensaios: $1.284 \mathrm{~kg} / \mathrm{cm}^{2}$

R. Fac. Odont. P. A. 


\subsubsection{Interpretação dos resultados:}

Os resultados encontrados demonstram respectivamente uma resistência à compressão de $1.625 \mathrm{~kg} / \mathrm{cm}^{2}$ para o silicato S. S. White e 1.284 $\mathrm{kg} / \mathrm{cm}^{2}$ para o silicato Astralit.

A diferença entre as duas médias é significativa ao nível de .01, indicando uma superioridade, neste sentido, do silicato S. S. White.

Embora ambos os materiais não preenchessem as normas, quanto à resistência à compressão, achamos, no entanto, que o resultado apresentado pelo material S. S. White muito se aproxima do mínimo exigido, ou seja: $1.700 \mathrm{~kg} / \mathrm{cm}^{2}$.

Submetemos então, os resultados à nova análise de variância, comparando os resultados individuais de cada marca, com o mínimo exigido pelas normas.

O resultado desta nova análise de variância nos demonstrou que a diferença entre os resultados obtidos com o silicato $\mathrm{S}$. S. White em nossos ensaios e o mínimo exigido segundo as normas, não é estatisticamente significativo ao nível de .01 .

O mesmo não sucedeu, entretanto, quando procedemos de maneira idêntica em relação ao silicato Astralit, em que encontramos que a diferenaa entre os resultados que obtivemos e o mínimo exigido pelas normas é estatisticamente significativa ao nivel de .01.

\section{RESUMO E CONCLUSÕES}

Os dois únicos cimentos de silicato de uso odontológico fabricados no Brasil (segundo nosso conhecimento) foram analisados, com o objetivo de verificar como se comportam face às normas estipuladas pela Federação Dentária Internacional e pela Associação Internacional de Padrões, normas estas aceitas pelo Grupo Brasileiro de Materiais Dentários.

De um modo geral, os silicatos em questão nos parecem satisfatórios, exceção feita à resistência à compressão, que nos pareceu bastante baixa no caso do silicato marca Astralit.

Esperamos que pesquisas desta natureza sirvam de incentivo e estimulem nossos fabricantes, no sentido de uma melhoria constante dos materiais dentários produzidos pela indústria nacional.

\section{S I N O P P I I S}

The properties, according to international specifications, of two dental silicate cements manufactured in Brazil, have been investigated. The products comply to the specifications, except as to compressive strenght valuse, which seemed particullarly low in one of the products. Dissemination of this type of information will not only assist tse dental profession in selecting proper materials, but will also encourage and stimulate the improvement in the manufacturing of dental materials. 
148

$\mathbf{x}_{1}=$ S. S. WHITE - Cor 21

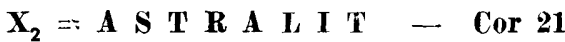

SOLUBILIDADE E DESINTEGRAÇa

$\begin{array}{lllllllllllllll}\text { T A A } & \text { E } & \text { L } & \text { A } & \text { D } & \text { E } & \text { C } & \mathbf{A} & \mathbf{L} & \mathbf{C} & \mathbf{U} & \mathbf{L} & \mathbf{O} & \mathbf{S}\end{array}$

\begin{tabular}{|c|c|c|c|c|c|c|c|}
\hline & $x_{1}$ & $\mathrm{X}^{2}{ }_{1}$ & $\mathrm{X}_{2}$ & $\mathrm{X}^{2}{ }_{2}$ & $\mathrm{x}_{3}$ & $\mathrm{X}_{3}{ }_{3}$ & $\mathrm{~S}$ \\
\hline 1 & 0,44 & 0,1936 & 0,62 & 0,3844 & & & \\
\hline 2 & 0,48 & 0,2304 & 0,84 & 0,7056 & & & \\
\hline 3 & 0,49 & 0,2401 & 0,86 & 0,7396 & & & \\
\hline 4 & 0,59 & 0,3481 & 0,87 & 0,7569 & & & \\
\hline 5 & 0,60 & 0,3600 & 1,18 & 1,3924 & & & \\
\hline 6 & & & & & & & \\
\hline 7 & & & & & & & \\
\hline 8 & & & & & & & \\
\hline 9 & & & & & & & \\
\hline 10 & & & & & & & \\
\hline$S x^{2}$ & & 7,7322 & & 3,9789 & & & 5,3511 \\
\hline Sx & 2,60 & & 4,37 & & & & 6,97 \\
\hline $\mathrm{r}$ & 5 & & 5 & & & & $\mathrm{n}=10$ \\
\hline $\bar{x}$ & 0,52 & & 0.874 & & & & $k=2$ \\
\hline
\end{tabular}

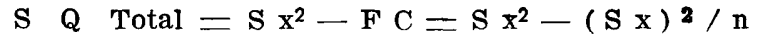

$\mathrm{S} \quad \mathrm{Q}$ Total $=5,3511-\mathrm{FC}=5,3511-4,85809=\mathbf{0 , 4 9 3 0 1}$

$\mathrm{S} \quad \mathrm{Q}$ Trat. $=\left(\mathrm{S} \mathrm{x}_{1}\right)^{2} / \mathrm{r}_{1}+\left(\mathrm{S} \mathrm{x}_{2}\right)^{2 / \mathrm{r}_{2}}+\left(\mathrm{S} \mathrm{x}_{3}\right)^{2 / \mathrm{r}_{3}}-\mathbf{F ~ C}$

$\mathrm{S} \quad \mathrm{Q} \quad$ Trat. $=1,352+3,81938-4,85809=\mathbf{0 , 3 1 3 2 9}$

\begin{tabular}{l|c|c|c|c}
\hline F O N T E & SO & SL & Q & Fc \\
\hline Tratamento & 0.31229 & $\mathrm{k}-1=1$ & 0,31329 & 13,948 \\
\hline E r r o & 0,17972 & $\mathrm{n}-\mathrm{k}=8$ & 0,02246 & \\
\hline $\mathrm{T} O \mathrm{~T} \mathrm{~A} \mathrm{~L}$ & 0.49301 & $\mathrm{n}-1=9$ & & \\
\hline
\end{tabular}

Ft .01; GL 1-8 = 11,26 LOGO: REJEITA-SE $\mathrm{H}_{0}$ As médias são diferentes. LUS/o10/atb

$05 / 71$

R. Fac. Odont. P. A. 


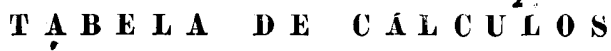

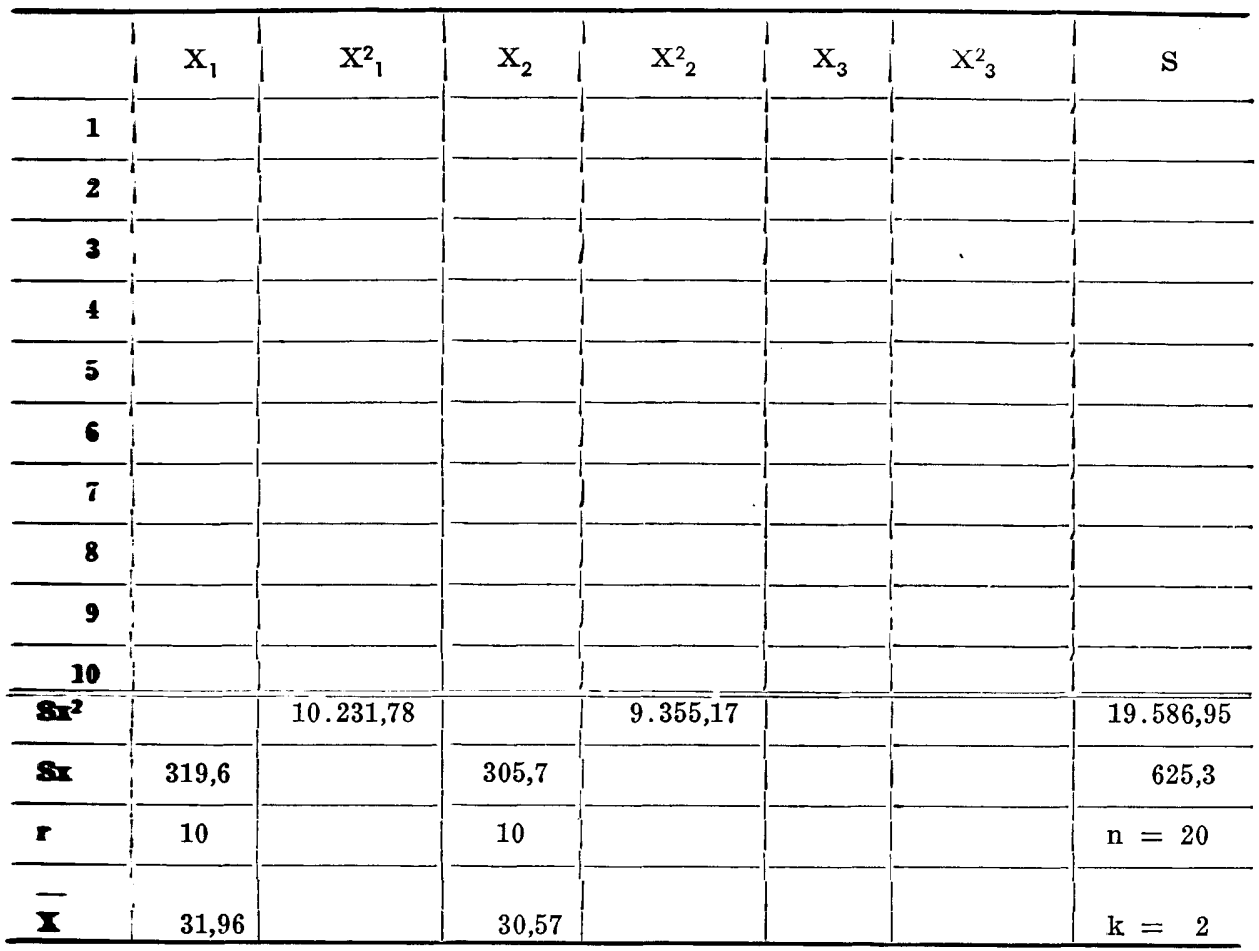

S Q Total $=\mathrm{S} \mathrm{x}^{2}-\mathrm{FC}=\mathrm{S} \mathrm{x}^{2}-(\mathrm{S} \mathrm{x})^{2} / \mathrm{n}$

s Q Total $=19.586,95-\mathrm{FC}=19.586,95-19.550,00=\mathbf{3 6 , 9 5}$

$\mathbf{s}$ Trat. $=\left(\mathrm{S} \mathrm{x}_{1}\right)^{2} / \mathrm{r}_{1}+\left(\mathrm{S} \mathrm{x}_{2}\right)^{2 / \mathrm{r}_{2}}+\left(\mathrm{S} \mathrm{x}_{3}\right)^{2} / \mathrm{r}_{3}-\mathrm{F} \mathrm{C}$

$\mathbf{8} \mathbf{Q}$ Trat. $=10.214+9.345-19.550=9$

\begin{tabular}{l|l|l|l|l}
\hline F O N T E & SQ & SL & Q & Fc \\
\hline Tratamento & 9,00 & $\mathrm{k}-1=1$ & 9,00 & 5,798 \\
\hline B r r o & 27,95 & $\mathrm{n}-\mathrm{k}=18$ & 1,552 & \\
\hline T O T A L & 36,95 & $\mathrm{n}-1=19$ & \\
\hline
\end{tabular}

In .01; GL $1-18=8,28$

LOS/o1o/atb

LOGO: ACEITASE $\mathrm{H}_{0}$

As médias são iguais.

$65 / 71$

R. Fac. Odont. P. A. 
150

$x_{1}=$ S. S. WHITE - Cor 21

$\mathbf{X}_{\mathbf{2}}=$ ASTRALIT Cor 21

RESISTENCIA A COMPRESSAO

TH A B E L L A D D E

\begin{tabular}{c|c|c|c|c|c|c|c}
\hline & $\mathrm{X}_{1}$ & $\mathrm{X}_{1}{ }_{1}$ & $\mathrm{X}_{2}$ & $\mathrm{X}_{2}{ }_{2}$ & $\mathrm{X}_{3}$ & $\mathrm{X}_{3}{ }_{3}$ & $\mathrm{~S}$ \\
\hline 1 & 1.605 & 2.576 .025 & 1.259 & 1.585 .081 & & & \\
\hline 2 & 1.521 & 2.313 .441 & 1.273 & 1.620 .529 & & & \\
\hline 3 & 1.804 & 3.254 .416 & 1.366 & 1.863 .225 & & & \\
\hline 4 & 1.641 & 2.692 .881 & 1.301 & 1.692 .601 & & & \\
\hline 5 & 1.556 & 2.421 .136 & 1.224 & 1.498 .176 & & & \\
\hline 6 & & & & & & & \\
\hline 7 & & & & & & & \\
\hline 8 & & & & & & & \\
\hline 9 & & & & & & & \\
\hline 10 & & & & & & & \\
\hline \hline $\mathrm{S} \mathrm{x}^{2}$ & & 13.257 .889 & & 8.259 .612 & & & 21.517 .511 \\
\hline $\mathrm{Sx}$ & 8.127 & & 6.422 & & & & 14.549 \\
\hline $\mathrm{r}$ & 5 & & 5 & & & & $\mathrm{n}=10$ \\
\hline$\overline{\mathrm{x}}$ & 1.625 & & 1.284 & & & & $\mathrm{k}=10$ \\
\hline
\end{tabular}

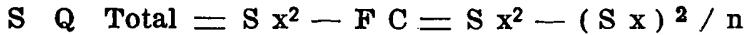

$\mathrm{S} \quad \mathrm{Q}$ Total $=21.517 .511-\mathrm{FC}=21.517 .511-21.167 .340=350171$

S $\quad \mathbf{Q}$ Trat. $=\left(\mathrm{S} \mathrm{x_{1 }}\right)^{2} / \mathrm{r}_{1}+\left(\mathbf{S} \mathrm{x}_{2}\right)^{2} / \mathrm{r}_{2}+\left(\mathbf{S} \mathrm{x}_{3}\right)^{2} / \mathrm{r}_{3}-\mathbf{F ~ C}$

$\mathrm{S} \quad \mathrm{Q}$ Trat. $=13.209 .625,800+8.248 .416,800-21.167 .340=290702,600$

\begin{tabular}{l|c|c|c|c}
\hline F O N T E & SQ & SL & Q & Fc \\
\hline Tratamento & $290.702,600$ & $\mathrm{k}-1=1$ & $290.702,600$ & 39,106 \\
\hline E r r o & $59.468,400$ & $\mathrm{n}-\mathrm{k}=8$ & $7.433,550$ & \\
\hline T O T A L & $350.171,000$ & $\mathrm{n}-1=9$ & & \\
\hline
\end{tabular}

Ft .01; GL 1-8 $=11,26 \quad$ LOGO: REJEITA-SE $\mathrm{H}_{0} \quad$ As médias são diferentes. LUS/o10/atb

$05 / 71$

R. Fac. Odont. P. A. 


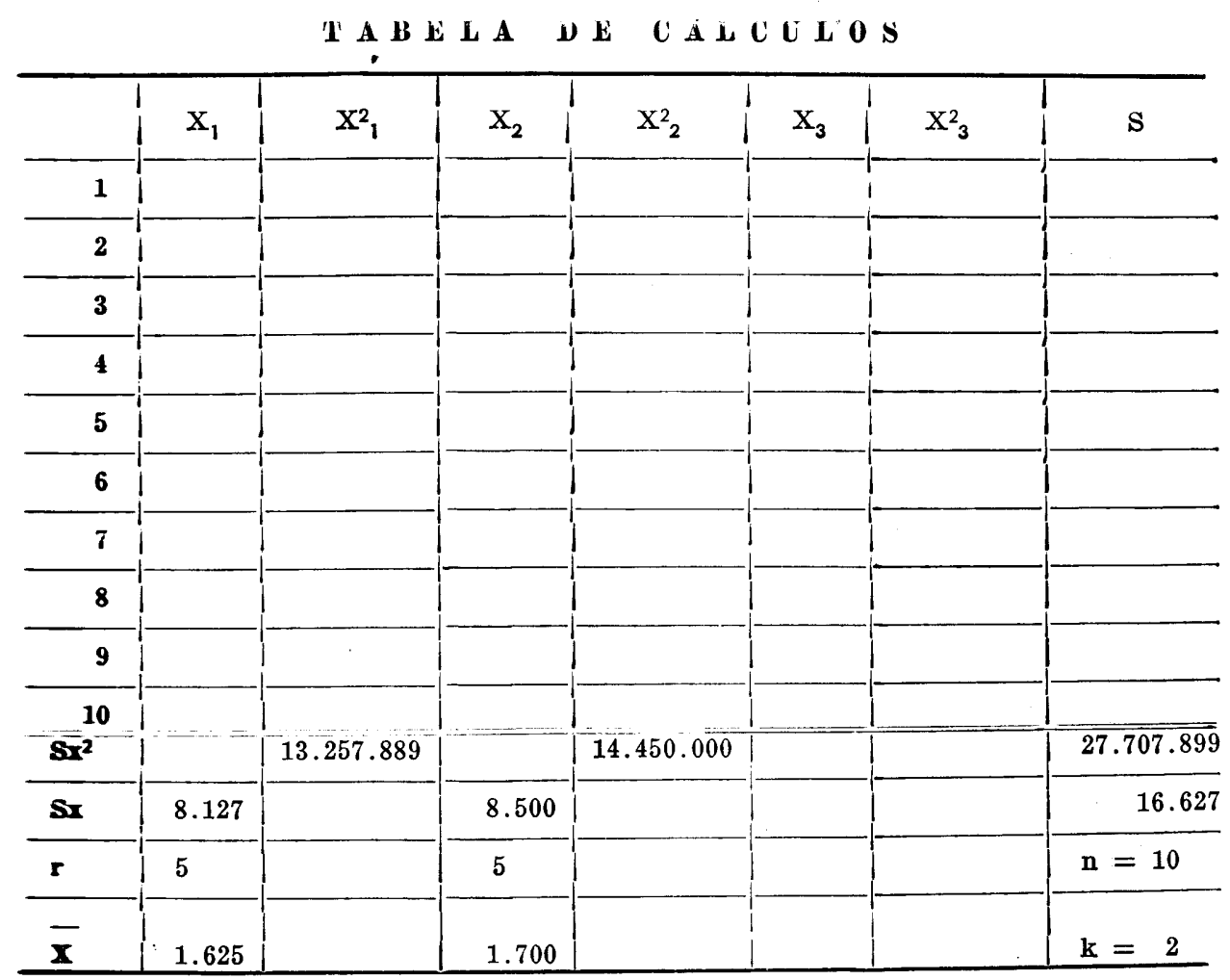

S $\mathbf{Q}$ Total $=\mathrm{S} \mathrm{x}^{2}-\mathrm{F} \mathrm{C}=\mathrm{S} \mathrm{x}^{2}-(\mathrm{S} \mathrm{x})^{2} / \mathrm{n}$

S $\mathbf{Q}$ Total $=27.707 .899-\mathrm{FC}=27.707 .899-27.645 .713=62.181$

$\mathbf{S} \mathbf{Q}$ Trat. $=\left(\mathrm{S} \mathrm{x}_{1}\right)^{2} / \mathrm{r}_{1}+\left(\mathrm{S} \mathrm{x}_{2}\right)^{2} / \mathrm{r}_{2}+\left(\mathrm{S} \mathrm{x}_{3}\right)^{2} / \mathrm{r}_{3}-\mathrm{F} \mathrm{C}$

$\mathbf{S} \mathbf{Q}$ Trat. $=13.209 .626+14.450 .000-27.645 .713=13.913$

\begin{tabular}{l|c|c|c|c}
\hline \multicolumn{1}{c|}{ F O N T E } & SQ & SL & Q & \multicolumn{1}{|c}{ Fc } \\
\hline Tratamento & 13.913 & $\mathrm{k}-1=1$ & 13.913 & 2,305 \\
\hline B r r o & 48.268 & $\mathrm{n}-\mathrm{k}=8$ & $6.033,500$ & \\
\hline T O T A L & 62.181 & $\mathrm{n}-1=9$ & & \\
\hline
\end{tabular}

I .01; GL 1-8 $=11,26$ LOGO: ACEITA-SE $H_{0}$ As médias são iguais. Les/o1o/atb

$\omega / 71$

R. Fac. Odont. P. A. 


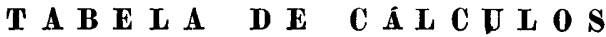

\begin{tabular}{|c|c|c|c|c|c|c|c|}
\hline & $x_{1}$ & $\mathrm{X}^{2}{ }_{1}$ & $\mathrm{X}^{2}$ & $\mathrm{X}^{2}{ }_{2}$ & $\mathrm{x}^{3}$ & $\mathrm{X}^{2}{ }_{3}$ & $\mathbf{s}$ \\
\hline 1 & & & & & & & \\
\hline 2 & & & & & & & \\
\hline 3 & & & & & & & \\
\hline 4 & & & & & & & \\
\hline 5 & & & & & & & \\
\hline 6 & & & & & & & \\
\hline 7 & & & & & & & \\
\hline 8 & & & & & & & \\
\hline s & & & & & & & \\
\hline 10 & & & & & & & \\
\hline $\mathrm{Sx}^{2}$ & & 8.259 .612 & & 14.450 .000 & & & 22.709 .612 \\
\hline $\mathbf{S x}$ & 6.422 & & 8.500 & & & & 14.922 \\
\hline $\mathbf{r}$ & 5 & & 5 & & & & $\mathrm{n}=10$ \\
\hline $\bar{x}$ & 1.284 & & 1.700 & & & & $\mathbf{k}=$ \\
\hline
\end{tabular}

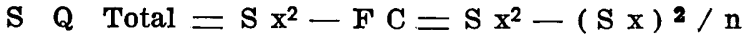

$\mathrm{S} \quad \mathrm{Q}$ Total $=22.709 .612-\mathrm{FC}=22.709 .612-22.266 .608=443.004$

S $\quad$ Q Trat. $=\left(\mathrm{S} \mathrm{x}_{1}\right)^{2} / \mathrm{r}_{1}+\left(\mathrm{S} \mathrm{x}_{2}\right)^{2} / \mathrm{r}_{2}+\left(\mathrm{S} \mathrm{x}_{3}\right)^{2} / \mathrm{r}_{3}-\mathrm{F} \mathrm{C}$

$\mathrm{S} \quad \mathrm{Q}$ Trat. $=8.248 .417+14.450 .000-22.266 .608=431809$

\begin{tabular}{l|c|c|c|c}
\hline \multicolumn{1}{c|}{ F O N T E } & SQ & SL & Q & Fc \\
\hline Tratamento & 431.809 & $\mathrm{k}-1=1$ & 431.809 & 308,655 \\
\hline E r r o & 11.195 & $\mathrm{n}-\mathrm{k}=8$ & 1.399 & \\
\hline T O T A L & 443.004 & $\mathrm{n}-1=9$ & \\
\hline
\end{tabular}

Ft .01; GL 1-8 $=11,26$ LOGO: REJEITA-SE $\mathrm{H}_{0} \quad$ As médias são diferentes. LUS/010/atb

$05 / 71$

R. Fac. Odont. P. A. 


\section{REFERÊNCIAS BIBLIOGRÁFICAS}

1. RAFFENBARGER, G.C. et alii - American Dental Association Specification no 9 for Dental Silicate Cement: First Revision, Effec. tive July 1, 1950. J.A.D.A. 40 (2) :186-193, Feb. 1950

2. COUNCIL ON DENTAL MATERIALS AND DEVICES, Guide to dental materials and devices, 4th Edition, 1968-69, American Dental Association, Chicago, USA, 1968 (p. 146-150)

3. GRUPO BRASILEIRO DE MATERIAIS DENTÁRIOS - Especificações Brasileiras no 3: Especificações para Cimentos de Silicato Odontológicos. Porto Alegre, 1965

4. FEDERATION DENTAIRE INTERNATIONALE - Specification for Dental Silicate Cement. Internatl. Dent. J. 11:536-543, Dec. 1961

5. INTERNATIONAL ORGANIZATION FOR STANDARDIZATION ISO, RECOMMENDATION R 1565 - Dental Silicate Cement, Ist Edition, June 1970.

6. SUFFERT, L.W. et alii - Indice de opacidade em cimentos de silicato: influencia da dimensão dos corpos de prova face à aparelhagem utilizada. Rev. Fac. Odont. UFRGS 13: 